DRAFT VERSION MARCH 21, 2022

Preprint typeset using LTEX style emulateapj v. 5/2/11

\title{
ANGULAR MOMENTUM FLUCTUATIONS IN THE CONVECTIVE HELIUM SHELL OF MASSIVE STARS
}

\author{
AVISHAI GILKIS AND NOAM SOKER \\ Department of Physics, Technion - Israel Institute of Technology, Haifa 32000, Israel; agilkis@tx.technion.ac.il; soker@physics.technion.ac.il \\ Draft version March 21, 2022
}

\begin{abstract}
We find significant fluctuations of angular momentum within the convective helium shell of a pre-collapse massive star - a core-collapse supernova progenitor - which may facilitate the formation of accretion disks and jets that can explode the star. The convective flow in our model of an evolved $M_{\mathrm{ZAMS}}=15 M_{\odot}$ star, computed with the sub-sonic hydrodynamic solver MAESTRO, contains entire shells with net angular momentum in different directions. This phenomenon may have important implications for the late evolutionary stages of massive stars, and for the dynamics of core-collapse.
\end{abstract}

Key words: stars: massive - supernovae: general

\section{INTRODUCTION}

Theory suggests that massive enough stars develop a core of iron-group elements, which upon reaching a critical mass undergoes a catastrophic collapse followed by an energetic and luminous explosion termed a core-collapse supernova (CCSN). Although stars are close to being spherical objects, there are phenomena for which a spherically symmetric onedimensional treatment may be insufficient, both in the nearly hydrostatic states during the long stellar lifetime prior to collapse, and in the hydrodynamic explosion itself.

In stellar evolution modeling, convective mixing and transfer of energy is a notably multi-dimensional phenomenon (e.g., Beeck et al. 2015). Convection is usually treated using the approximate semi-empirical Mixing-Length Theory (MLT). This simplified treatment might be inadequate in some cases. Ongoing studies aim at improving the theoretical modeling and understanding of convection in stellar interiors (e.g., Meakin \& Arnett 2007; Arnett et. al 2009, 2015; Viallet et al. 2013, 2015, 2016).

Examining explosion models, the long-studied neutrinodriven mechanism (Colgate \& White 1966; Bethe \& Wilson 1985) fails in most one-dimensional simulations (e.g., Burrows, Hayes \& Fryxell 1995; Rampp \& Janka 2000; Mezzacappa et al. 2001; Liebendörfer et al. 2005), and it might be important to look at multi-dimensional effects such as the standing accretion-shock instability (SASI; e.g., Blondin, Mezzacappa \& DeMarino 2003; Blondin \& Mezzacappa 2007; Fernández 2010). Papish, Nordhaus \& Soker (2015) suggest that neutrinodriven mechanisms might not work.

The multi-dimensional phenomena in evolution and explosion modeling may be interconnected. Recent studies (Couch \& Ott 2013, 2015; Mueller \& Janka 2015) found that non-spherical perturbations in the progenitor may increase post-collapse turbulent pressure, significantly influencing the dynamic behavior in the shocked region surrounding the forming NS. In these studies, large velocity fluctuations were introduced in the pre-collapse core. In an earlier paper (Gilkis \& Soker 2015) we found that such velocity perturbations imply sufficiently large specific angular momentum fluctuations for the formation of intermittent accretion disks and a jittering-jets explosion.

Among the alternatives to the neutrino-driven explosion mechanism (see Janka 2012 for a review), most promi- nent are jet-driven explosions (e.g., LeBlanc \& Wilson 1970; Khokhlov et al. 1999; Lazzati et al. 2012). Another interesting mechanism is the collapse-induced thermonuclear explosion (Kushnir \& Katz 2015; Kushnir 2015a b). The jetdriven scenario is inherently multi-dimensional, where a pre-collapse rapid core rotation may facilitate the formation of magnetorotationally driven bipolar outflows (Mösta et al. 2015). We note that the above cited works require a rapidly rotating pre-collapse core, and hence limited to a small fraction of all CCSNe.

A different approach of jet-driven explosions tries to explain all energetic CCSNe, for cases of rapid as well as slow stellar rotation (Soker 2010; Papish \& Soker 2011, 2012a b, 2014a b). In this model accretion from material with varying angular momentum in a collapsing star leads to the formation of accretion disks with varying axis direction. These intermittent accretion disks launch jets with different directions, termed 'jittering jets', that might explode the star with the observed energy. The jittering jets model faces the challenge of supplying the required angular momentum for an accretion disk, and consequently forming jets. The required angular momentum for jet formation may be lower than usually implied, when a dynamo operates in a shear layer around the proto-neutron star (Akiyama et al.2003; Schreier \& Soker 2016).

Recent studies have concentrated on non-spherical perturbations in the close vicinity of the iron core just prior to collapse (e.g., Couch et al. 2015). We look a bit earlier in the stellar evolution and further out in the star for a possible source of angular momentum. While the outer hydrogen region probably has tremendous fluctuations of angular momentum, the sudden change in the gravitational potential as a result of neutrino emission during NS formation, results in the expulsion of the envelope (Lovegrove \& Woosley 2013). Analytic estimations show that the convective helium shell of an evolved massive star may have large fluctuations of angular momentum as well (Gilkis \& Soker 2014). In a scenario where after core-collapse the inner silicon/oxygen regions fail to produce a sufficient driving force for a supernova explosion, accretion from the helium region might result in the formation of intermittent accretion disks and jets. Accretion from material with varying angular momentum in a collapsing star can lead to an energetic supernova explosion in the jittering jets model.

In the present study we explore the properties of the pre- 


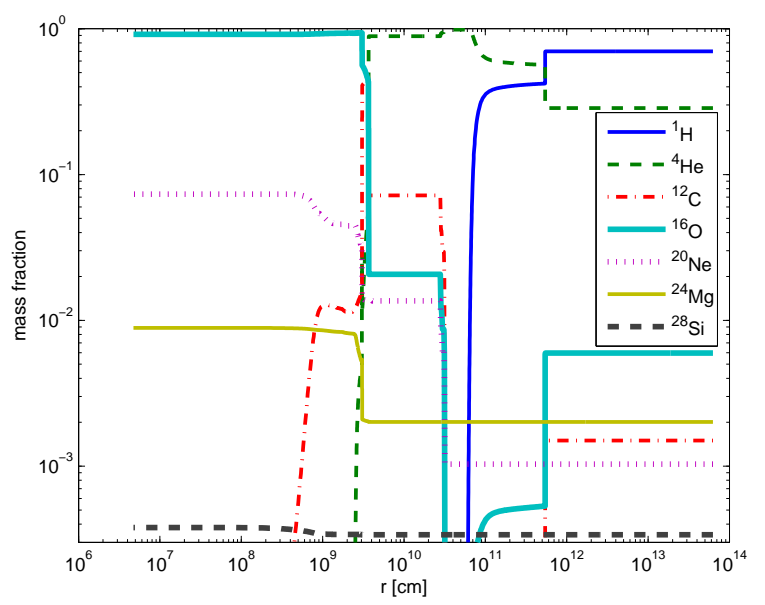

FIG. 1. - Detailed composition for our stellar model of a non-rotating $M_{\mathrm{ZAMS}}=15 M_{\odot}$ star with metallicity of $Z=0.014$. The profile is presented at an age of $t=12.5 \mathrm{Myr}$, about $5.5 \mathrm{yr}$ before explosion. The stellar parameters at this stage are as follows. Stellar mass of $M=13.3 M_{\odot}$, effective temperature of $T=3235 K$, photospheric radius of $R=902 R_{\odot}$ and luminosity of $L=8 \times 10^{4} L \odot$. The convective helium shell simulated here extends from $r_{\text {in }}=3.7 \times 10^{9} \mathrm{~cm}$ to $r_{\text {out }}=2.8 \times 10^{10} \mathrm{~cm}$. Results below will all be presented at this time and for this model.

collapse convective helium region. We perform a threedimensional hydrodynamic simulation of the convective flow, strengthening our preliminary analytic estimations. The helium region does not change much in the relatively short time left until collapse, so that our results are relevant for the final stage of the stellar evolution. In section 2 we describe the numerical setup and method. Our main results are presented in section 3 . Some implications for core-collapse are concisely discussed in section 4. We summarize in section 5 .

\section{NUMERICAL SETUP}

We use a stellar model constructed by Modules for Experiments in Stellar Astrophysics (MESA version 5819; Paxton et al. 2011, 2013), with an initial mass of $M_{\mathrm{ZAMS}}=$ $15 M_{\odot}$ and metallicity of $Z=0.014$. Due to stellar winds calculated here with the so-called 'Dutch' scheme (e.g., Nugis \& Lamers 2000; Vink, de Koter \& Lamers 2001) the final mass is $13.3 M_{\odot}$. Effects of rotation and magnetic fields are neglected. We examine the star during the helium shell burning stage, about 5.5 years prior to collapse. At this stage, the star is a red supergiant (RSG) with an effective surface temperature of $T=3235 \mathrm{~K}$, photospheric radius of $R=902 R_{\odot}$ and luminosity of $L=8 \times 10^{4} L_{\odot}$. The detailed composition of the stellar model is shown in Figure 1 .

There are two reasons for simulating the star at this time. (i) The convective helium shell may be of interest as a potential supply of material with angular momentum for accretion onto the newly-formed compact object following corecollapse, in a scenario where the inner shells fail in driving a successful explosion. The convective hydrogen envelope is less relevant, as it is likely to be expelled due to decrease of the NS gravitational mass by neutrino cooling (Nadezhin 1980; Lovegrove \& Woosley 2013). The structure outside $r_{\mathrm{O}-\text { core }} \approx 3.5 \times 10^{9} \mathrm{~cm}$ remains essentially unchanged until core-collapse, so the results presented here are relevant for the core-collapse epoch. If we had taken an earlier point of the stellar evolution, the different core structure would have lessened the pertinence of our simulation. (ii) While in the final stage before core-collapse there are several convective shells (silicon and oxygen, in addition to helium and hydrogen at the simulated stage), the complex structure would have made the simulation more difficult to run. We hope the methods detailed here will inspire future works on angular momentum fluctuations in the silicon and oxygen shells in core-collapse supernova progenitors.

Convection in MESA is according to the Mixing-Length Theory (MLT), which gives for our model a convective outer hydrogen envelope, and a convective helium region surrounding a radiative oxygen core. The outer part of the helium region is radiative as well. In this study we are not interested in the outer envelope, and focus on the convective helium region, extending from $r_{\text {in }}=3.7 \times 10^{9} \mathrm{~cm}$ and $M_{\text {in }}=2.4 M_{\odot}$, to $r_{\text {out }}=2.8 \times 10^{10} \mathrm{~cm}$ and $M_{\text {out }}=3.7 M_{\odot}$. If and when this region collapses to the core, the free fall time from the inner and outer boundaries of the helium convective regions are $t_{\mathrm{ff}-\text { in }}=14 \mathrm{~s}$, and $t_{\mathrm{ff}-\text { out }}=228 \mathrm{~s}$, respectively. Interestingly, this range is compatible with the duration of long gamma ray bursts (LGRB). This is not the case studied here, but the behavior of the helium convective layer might be relevant to the formation of jets in GRBs.

The inner region of the one-dimensional MESA model is mapped into a three-dimensional grid in the low Mach number stellar hydrodynamics code MAESTRO (Nonaka et al. 2010). The MAESTRO code was developed mainly for the purpose of studying convection in white dwarfs experiencing thermonuclear deflagration (Almgren et al. 2006a.b, 2008; Zingale et al. 2009), though more recently also for modeling convection preceding Type I X-ray Bursts (Malone et al. 2011, 2014; Zingale et al. 2015) and convection in massive stars (Gilet et al. 2013). This latter study motivated us to use MAESTRO for our purpose - the study of angular momentum fluctuations in a convective region of a massive star. For this purpose we need a full sphere domain, with no imposed reflective boundaries for reduction of computational costs.

Similarly to Gilet et al. (2013), we initialize the velocity field with small perturbations (much lower in magnitude than the expected, and obtained, flow velocities), and use velocity damping near the edges of the simulation domain (far from the regions of interest). We further added velocity damping in the oxygen core, as we encountered numerical problems in this region of the simulation - spurious velocities and heating. This may be due to the absence of radiative transfer in the simulation (important in the non-convective core) or because of insufficient resolution. We ran simulations with damping of the entire core, part of it, and no damping at all. The simulated convective helium region changed little between the simulations, and the inner oxygen core is of no particular interest in the present study. We therefore conclude that our results are robust, although we may need to address this issue in a future work.

We have incorporated an approximate reaction network in the code, which includes only the triple-alpha process (the only relevant process in the region of interest). The energy generation rate is according to Kippenhahn, Weigert \& Weis 
(2012), p. 197,

$$
\begin{aligned}
& \epsilon_{3 \alpha}=6.272 \rho^{2} X_{4}^{3} \cdot\left(1+0.00158 T_{9}^{-0.65}\right) \\
& \times\left[2.43 \times 10^{9} T_{9}^{-2 / 3} \exp \left(-13.490 T_{9}^{-1 / 3}-\left(T_{9} / 0.15\right)^{2}\right)\right. \\
& \left.\times\left(1+74.5 T_{9}\right)+6.09 \times 10^{5} T_{9}^{-3 / 2} \exp \left(-1.054 / T_{9}\right)\right] \\
& \times\left[2.76 \times 10^{7} T_{9}^{-2 / 3} \exp \left(-23.570 T_{9}^{-1 / 3}-\left(T_{9} / 0.4\right)^{2}\right)\right. \\
& \times\left(1+5.47 T_{9}+326 T_{9}^{2}\right)+130.7 T_{9}^{-3 / 2} \exp \left(-3.338 / T_{9}\right) \\
& \left.+2.51 \times 10^{4} T_{9}^{-3 / 2} \exp \left(-20.307 / T_{9}\right)\right],
\end{aligned}
$$

where $\epsilon_{3 \alpha}$ is in erg $\mathrm{gr}^{-1} \mathrm{~s}^{-1}, \rho$ is the density, $X_{4}$ is the helium mass fraction, and $T_{9}$ is the temperature divided by $10^{9} \mathrm{~K}$. We have neglected composition changes, as the nuclear timescale is significantly longer than the dynamical timescale.

The Adaptive Mesh Refinement (AMR) capabilities of MAESTRO were employed to keep a grid resolution better than $0.1 H_{P}$ throughout the convective helium region, where $H_{P}$ is the pressure scale height. We performed several simulations with lower finest resolution (higher resolutions are prohibited with our current computational resources). The magnitude of the angular momentum, which was our primary concern, is similar in the different simulations. The maximal Mach number of the convective motion and the average velocity, however, increase with decreasing resolution. Clearly, simulations with higher resolution are required to fully understand the phenomena investigated here, but for this introductory study our results are sufficient. A detailed analysis of the lower-resolution full simulations is presented in Appendix A and additional simulations of one eighth of the domain and higher resolution are presented in Appendix B.

\section{SIMULATION RESULTS}

\subsection{Angular momentum}

We ran the simulation for a physical time of close to $t=$ $20000 \mathrm{~s}$. This is about 85 times the free fall time from the outer convective boundary $t_{\mathrm{ff}-\mathrm{out}}$, and about 8 times the typical convective overturn time $t_{\text {conv }} \equiv D_{\text {conv }} / v_{\text {conv }} \approx 2500 \mathrm{~s}$. The latter is calculated from the the typical diameter of the vortices $D_{\text {conv }} \approx 5 \times 10^{9} \mathrm{~cm}$, and the typical velocity in the vortices of $v_{\text {conv }} \approx 2 \times 10^{6} \mathrm{~cm} \mathrm{~s}^{-1}$, as seen in figures 2 and 3 presented at the end of the simulation. Figure 2 shows the flow morphology, with color indicating the local specific angular momentum calculated from the flow velocity. Figure 3 shows a close-up of a region from Figure 2, with color indicating the magnitude and sense of the velocity, to emphasize the large-scale vortices that develop in the core.

Figure 4 shows the evolution with time of the global computational diagnostics of the maximal Mach number and the averaged velocity magnitude. It can be seen that after leaving the initial close-to-zero velocity setup, a steady flow develops after a time of about $t_{\text {conv }}$. Another important conclusion drawn from this figure is that the typical convective velocities are much higher than values given by the mixing-length theory. Similar results of higher than the MLT velocities have been obtained by, e.g., Arnett et. al (2009). This result holds even when a velocity lower by a factor of 2 is taken, as might be the case with higher resolution (see Appendix B).

Figure 5] shows the evolution of the angular momentum in several shells. We notice the significant net angular momen-

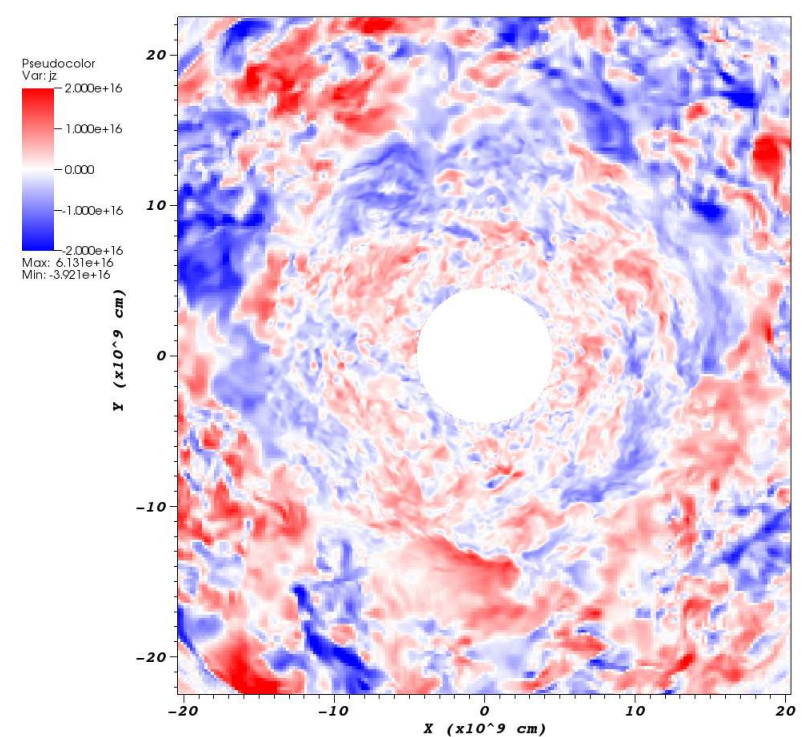

FIG. 2.- Specific angular momentum in the $x y$-plane at $t=20000 \mathrm{~s}$ in the simulation. Blue-shaded regions have a clockwise azimuthal velocity component $\left(j_{z}<0\right)$, while red-shaded regions have a counterclockwise azimuthal velocity component $\left(j_{z}>0\right)$. White regions have no azimuthal velocity component $\left(j_{z}=0\right)$. The color-coding runs from $-2 \times 10^{16} \mathrm{~cm}^{2} \mathrm{~s}^{-1}$ up to $2 \times 10^{16} \mathrm{~cm}^{2} \mathrm{~s}^{-1}$, although the specific angular momentum exceeds this value at some points. The inner oxygen core is omitted from the figure.

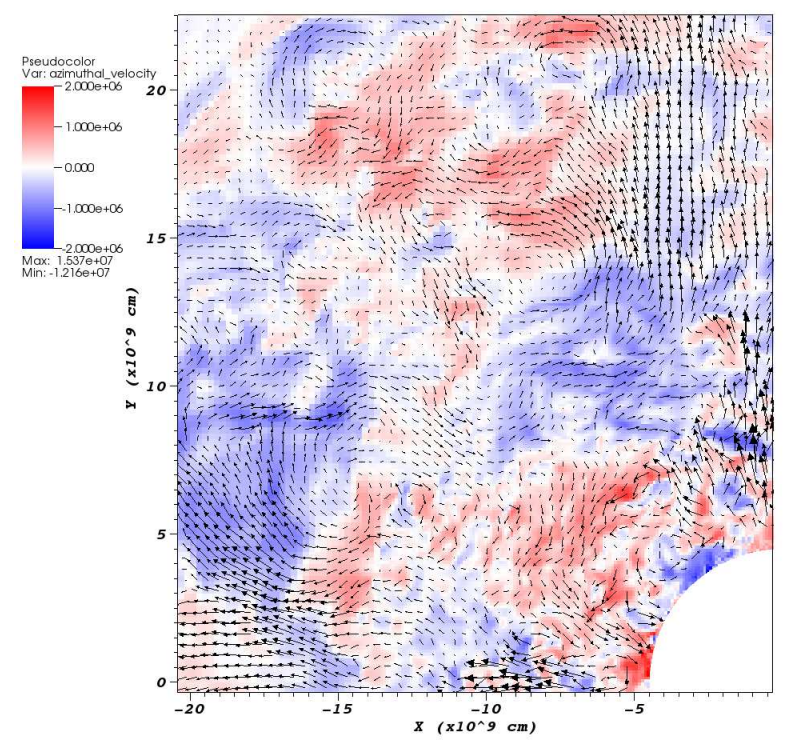

FIG. 3.- Azimuthal velocity color-map for a close-up of a region from Figure 2 with added velocity arrows for illustration of the vortices in the flow. The color-coding runs from an azimuthal velocity of $-2 \times 10^{6} \mathrm{~cm} \mathrm{~s}^{-1}$ up to $2 \times 10^{6} \mathrm{~cm} \mathrm{~s}^{-1}$. Similarly to Figure 2 blue-shaded and red-shaded regions have clockwise and counterclockwise velocity components, respectively. The velocity arrows are scaled - larger arrows represent higher velocities (an arrow spanning $10^{9} \mathrm{~cm}$ on the plot, for example, represents a velocity of $\left.2 \times 10^{6} \mathrm{~cm} \mathrm{~s}^{-1}\right)$. 

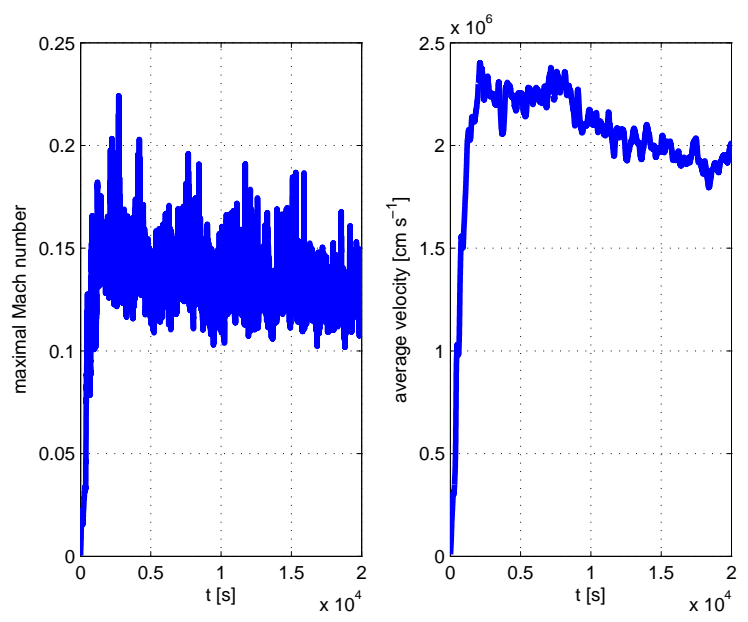

FIG. 4.- Left: Maximal Mach number in the simulation domain. The values reached in the simulation, of $\mathcal{M} \approx 0.15$, are two orders of magnitude above the mixing-length theory value for this model $\left(\mathcal{M}_{\mathrm{MLT}} \approx 10^{-3}\right)$. Right: Average velocity in the simulation domain. This too is much higher than the velocities of the MLT, which are around $v_{\mathrm{MLT}} \approx 10^{5}-2 \times$ $10^{5} \mathrm{~cm} \mathrm{~s}^{-1}$ for our stellar model. The disparity to the MLT is smaller for the averaged velocity, as there is an extensive range of velocities in the flow field, and the maximal velocities (and Mach numbers) reach values significantly higher than the mean.

tum that is developed in the shells, and the varying direction of the angular momentum axis. In Figure 6 we show the specific angular momentum profile of the material within the inner part of the convective region at $t=20000 \mathrm{~s}$ in the simulation. In plotting Figure 6 we used shells of width $\Delta r=2000 \mathrm{~km}$, much thinner than the $\Delta r=40000 \mathrm{~km}$ shells used in drawing Figure 5 The difference in free fall times between the two boundaries of the thin shells is $t_{f f} \approx 1-3 \mathrm{~s}$, equivalent to thousands of dynamical times on the surface of the newly formed NS (or BH). Shorter time scales may be of interest for the post-collapse dynamics, but a higher resolution is required, accordingly.

From Figure 4 we note that the average convective velocity increases rapidly within the first $2 \times 10^{3} \mathrm{~s}$. The value then decreases and increases back, followed by a decrease in the time interval $8 \times 10^{3}-1.8 \times 10^{4} \mathrm{~s}$, after which it increases until we terminate the simulation. Variations over a timescale of $\approx 0.5-1 \times 10^{4} \mathrm{~s}$ are seen also in the values of the angular momentum in thick shells as presented in Figure 5 Both figures 4 and 5 seem to suggest that the simulation has reached a more or less steady state, but with significant fluctuations. It would be useful to extend the calculations to check that this is actually the case, although this is computationally expensive. The large amplitudes and the long timescale of the fluctuations are the source of the relatively large value of the stochastic specific angular momentum of the accreted gas.

Another indication that we have reached a more or less steady state is presented in Figure 6 We see that the specific angular momentum components in thin shells reach maximum values that are about equal to those of the standard deviation of specific angular momentum calculated analytically from the MLT as in Gilkis \& Soker (2014). This estimation depends on the shell width, $\Delta r$, which in the present work is larger than in Gilkis \& Soker (2014). In our previous work, the thin shells had a width equivalent to a difference of freefall times that is a small multiple of the Keplerian time for a NS, which explains the higher values obtained for the standard deviation of specific angular momentum there. Addition- ally, the analytic estimation using MLT values for the present work is somewhat problematic, as the flow velocity is higher in the numerical simulation than in MLT. On the other hand, the length scale taken may need to be smaller than the mixinglength, which is close to the scale of vortices here, while the relevant length might be only part of a vortex. These factors compensate each other to some extent, so that the analytic estimation is close to the fluctuations of specific angular momentum in the simulation. This argument is an orderof-magnitude one, but nonetheless supports the claim that a steady state has been achieved. The diameter of the vortex, for example, is taken to be of the order of the mixing-length (or the radius of the vortex $a_{c}$ about equal to half the mixinglength). This order of magnitude estimate, as presented in Figure 6 further demonstrates the merit of the analytic model.

\subsection{Convective luminosity}

To further reveal the convective properties of the 3D simulations we study now the energy transport property of the convective motion. We start by writing the three equations assumed by the MLT. In doing so we follow the nomenclature of Kippenhahn et al. (2012). The convective flux is given by the heat capacity per unit mass of the gas $c_{p}$ multiplied by the mass flux $\rho v_{c}$, where $v_{c}$ is the convective velocity according to the MLT, and by the temperature difference $D T$ between the starting and end point of the convective element

$$
F_{\text {conv }}=\rho v_{\mathrm{c}} c_{p} D T \text {. }
$$

The temperature difference is given by

$$
D T=\left(\nabla-\nabla_{e}\right) \frac{l_{m}}{2} \frac{1}{H_{p}} T
$$

where $\nabla \equiv \partial \ln T / \partial \ln P$ is the temperature gradient (with respect to pressure) in the star, while $\nabla_{e}$ is the temperature gradient of the convective element; $l_{m}$ is the mixing length and $H_{p}$ is the pressure scale height. The velocity of the convective element is given by

$$
v_{\mathrm{c}}^{2}=g \delta\left(\nabla-\nabla_{e}\right) \frac{l_{m}^{2}}{8 H_{p}},
$$

where $\delta \equiv-(\partial \ln \rho / \partial \ln T)_{P}$; for an ideal gas $\delta=1$.

We now manipulate these three equations as follows. From equations (3) and (4) we derive

$$
D T=\frac{4 T}{g \delta l_{m}} v_{\mathrm{c}}^{2}
$$

which together with equation (2) give

$$
F_{\text {conv }}=\rho v_{\mathrm{c}}^{3} \frac{4 T c_{P}}{g \delta l_{m}} .
$$

The convective luminosity, equals to the integration of the convective flux over a spherical surface, can be written then in the form

$$
L_{\mathrm{conv}}=\Lambda(r) L_{\mathrm{KE}},
$$

where $\Lambda(r) \equiv 4 T c_{P} / g \delta l_{m}$ depends only on the radius. In the MLT the kinetic luminosity $L_{\mathrm{KE}}$ is given by

$$
L_{\mathrm{KE}-\mathrm{MLT}}(r) \equiv 4 \pi r^{2} \rho v_{\mathrm{c}}^{3} \text {. }
$$

Let us summarize the important assumptions that went into the derivation of equation (8). (i) The convective elements perform radial motion. (ii) They are destroyed at a radius that 

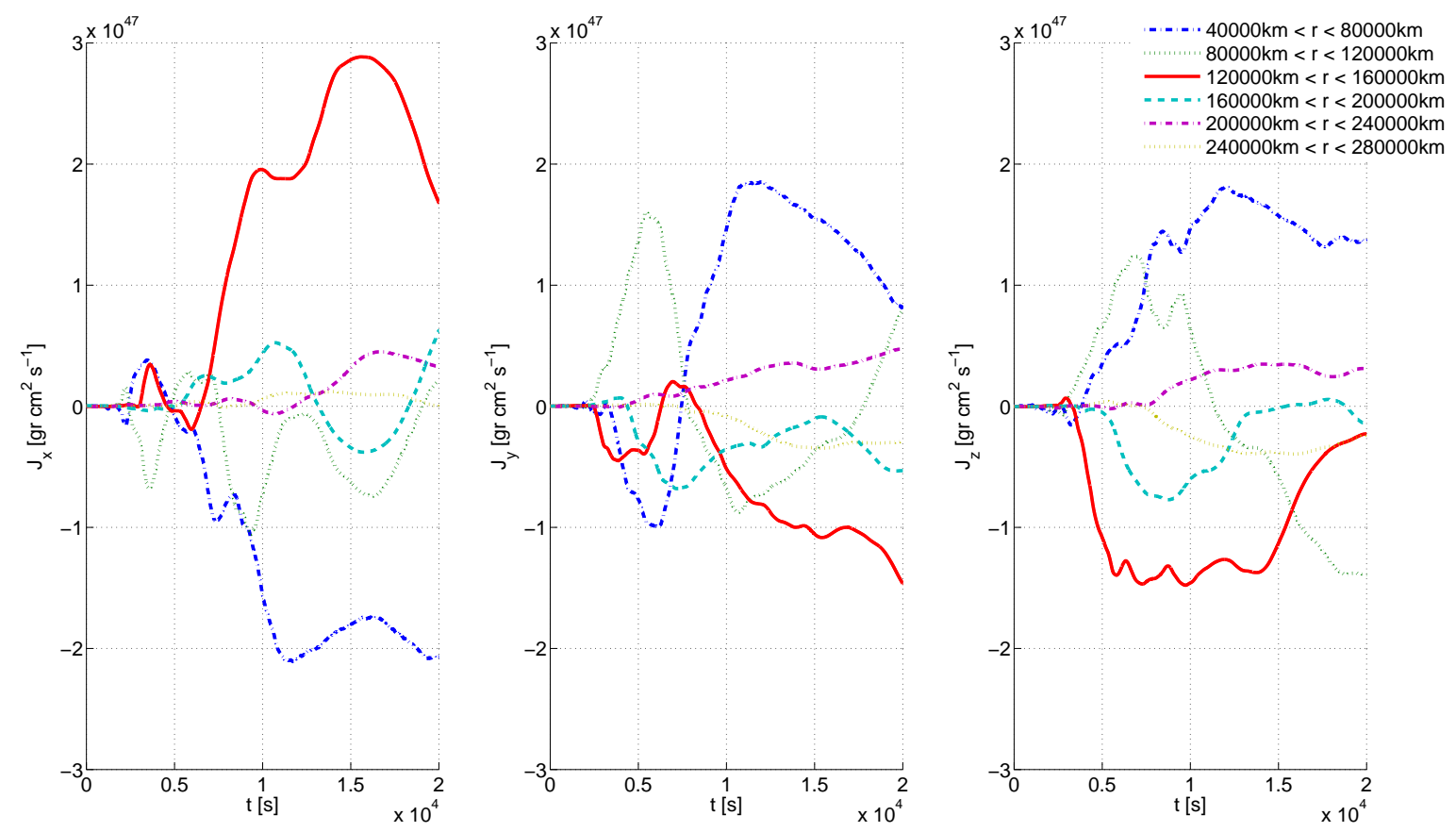

FIG. 5.- Angular momentum components of several shells within the convective region throughout the simulation time. The location of the shells is indicated in the inset in the right panel. Several shells possess net angular momentum with large values and in different directions. The masses of the shells from the inner shell to the outer are $0.25,0.25,0.23,0.22,0.19$ and $0.17 M_{\odot}$. The core mass inner to the helium shell is $M_{\mathrm{in}}=2.4 M_{\odot}$. The average specific angular momentum is therefore approximately few $\times 10^{14} \mathrm{~cm}^{2} \mathrm{~s}^{-1}$, which is lower than the specific angular momentum for a Keplerian orbit around a NS or BH. However, the partition into shells with widths of $\Delta r=40000 \mathrm{~km}$ is arbitrary. While we see here the large-scale behavior, our simulation might not capture some finer details relevant for post-collapse dynamics, as the differences in the free-fall times between the shell boundaries correspond to thousands of orbital times around the newly-formed NS or BH. A more focused analysis of the angular momentum distribution within shells at one time is shown in Figure 6

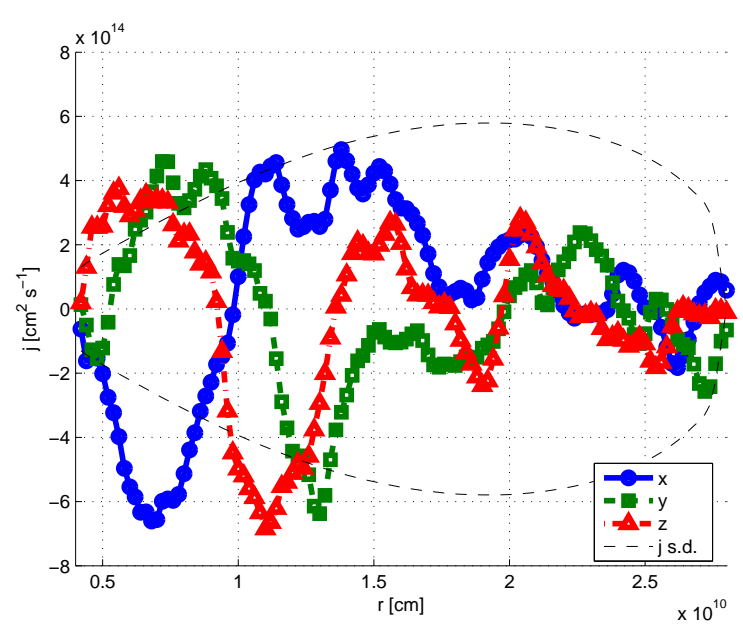

FIG. 6.- Specific angular momentum components in thins shells of $\Delta r=2000 \mathrm{~km}$ at $t=20000 \mathrm{~s}$ in the convective zone in the simulation, between $r=40000 \mathrm{~km}$ and $r=280000 \mathrm{~km}$. Black dashed lines show an analytic approximation of the standard deviation of specific angular momentum in the MLT, following Gilkis \& Soker (2014) so that $\sigma(j)=(2 / 27)^{1 / 2}(\Delta r)^{-1 / 2} a_{c}^{3 / 2} v_{c}$, with $a_{c}$ taken as half the mixinglength and $v_{c}$ the MLT convection velocity. The specific angular momentum for a Keplerian orbit around a newly-formed NS or BH is approximately $j_{\text {Kep }} \approx$ few $\times 10^{16} \mathrm{~cm}^{2} \mathrm{~s}^{-1}$; the values for the thin shells shown here is lower, reaching $j_{\max }$ (fluctuations) $\approx 6.85 \times 10^{14} \mathrm{~cm}^{2} \mathrm{~s}^{-1} \approx$ $0.03 j_{\mathrm{Kep}}$. Although the specific angular momentum is lower than the Keplerian, it is not negligible, and a belt-like structure might form around the NS or BH (as discussed by Papish, Gilkis \& Soker 2015). The difference in free-fall times for $\Delta r=2000 \mathrm{~km}$ is $1-3 \mathrm{~s}$, corresponding to hundreds of orbital times around the newly-formed compact object, so there is sufficient time to form accretion belts and jets upon core-collapse. is larger by $l_{m}$ from where they were formed. (iii) They transfer all their extra thermal energy where they are destroyed.

In the full 3D simulations we find that the motion is composed of vortices, rather than radial motions (Fig. 3). This implies that the hotter gas that is flowing at the top of a vortex loop is not destroyed, hence does not transfer to the surroundings all of its thermal energy. However, the net flux should be about equal to that in the MLT, as the structure of the star is the same, hence same luminosity. To compare the convective luminosity in our 3D simulation to that of the MLT, we need to replace $L_{\mathrm{KE}-\mathrm{MLT}}$ as given in eqaution (8) by

$$
L_{\mathrm{KE}-3 \mathrm{D}}(r)=r^{2} \int_{0}^{\pi} \sin \theta d \theta \int_{0}^{2 \pi} d \phi \rho v^{2}(\vec{v} \cdot \hat{r}) .
$$

where the density and velocity now depend on the location, $\rho(r, \theta, \phi)$ and $\vec{v}(r, \theta, \phi)$, respectively. To take into account the numerical grid, in calculating $L_{\mathrm{KE}-3 \mathrm{D}}(r)$ equation (9) is replaced by

$$
\begin{aligned}
& L_{\mathrm{KE}-3 \mathrm{D}}(r)=\frac{1}{\Delta r} r^{2} \\
& \quad \times \int_{r}^{r+\Delta r} d r^{\prime} \int_{0}^{\pi} \sin \theta d \theta \int_{0}^{2 \pi} d \phi \rho v^{2}\left(\vec{v} \cdot \hat{r}^{\prime}\right) .
\end{aligned}
$$

In Figure 7 we plot the kinetic luminosity of the MLT according to equation (8), in blue dots, and that of the full 3D simulation according to equation (10), in green squares. Although the kinetic luminosity of the 3D simulation is not identical to that of the MLT, it has the same bulk behavior. Our results at other times show a kinetic luminosity profile 


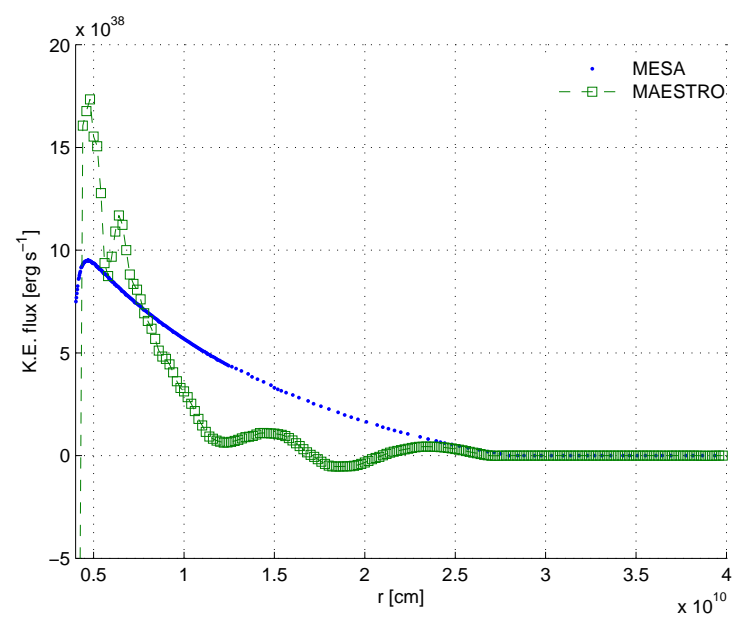

FIG. 7.- Comparing the kinetic luminosity of the MLT according to equation 8), in blue dots with that of the full 3D simulation according to equation [10, in green squares.

that changes with time. For example, the peak near the inner boundary changes slightly, such that the average value of the $3 \mathrm{D}$ results is less than twice that of the MLT near the peak of the kinetic luminosity. Overall, Figure 7 makes us confident in the convective properties revealed by our 3D simulations. Most important, the convective region is composed of large vortices that may translate after collapse to local fluctuations in the specific angular momentum of the mass accreted by the newly born NS or BH.

Despite our finding that the typical convective velocity in the 3D simulation is much larger than the typical velocity in the MLT, the convective luminosity of the two cases are very similar. The reason is as follows. In the MLT the convective velocity is calculated from the assumption that hot convective cells deposit all their extra energy after they move a radial distance equal to the mixing-length. This implies that the calculation of the convective luminosity of the MLT involves only upward moving gas. In the full 3D simulations convective cells are not destroyed when they reach maximum radius in the convective zone. They rather perform motions along vortices, and deposit a small fraction of their extra energy when they reach the maximum radius in their cyclical motion. Therefore, the integral performed in equation (9) (or eq. 10) involves a large flux downward. Namely, the factor $\vec{v} \cdot \hat{r}$ in those equations posses both positive (for upward motion) and negative (for downward motion) values. The very large flux upward minus the very large flux downward gives a value of the convective luminosity about equal to that in the MLT, as expected if the numerical simulation converges to a solution that yields the correct luminosity through the convective zone.

\section{IMPLICATIONS FOR CORE-COLLAPSE}

The flow structure of the helium presented in section 3 is probably similar to that just prior to collapse, and we can look at possible implications on the post-collapse dynamics. The scale of the vortices is such that the difference between the free-fall times onto the newly formed NS or black hole $(\mathrm{BH})$, between the edges of a vortex, is about tens of seconds. The local specific angular momentum as shown in Figure 2 is higher than the Keplerian specific angular momentum for orbit around a newly-formed NS. The large vortices imply then that on a short time scale of $\approx 0.01 \mathrm{~s}$, equal to several dynam- ical times on the newly born NS or BH, a small number of vortices contribute to the accretion, and only a small fraction from a vortex is accreted, contributing a small mass with high specific angular momentum. The differences in free-fall times between shells with differing angular momentum axes, equivalent to many orbital times close to compact object (where we assume jets are launched), allow for sufficient time for formation of disks and jets. The average value obtained for shells as described in Figure 6 is lower, and may lead to the formation of a thick accretion disk (or an accretion belt; for further discussion of this general effect see Gilkis \& Soker 2015 and Papish, Gilkis \& Soker 2015).

Considering jittering jets, which we suggest explode the star, we can make the following crude energy estimate. We take only shells with the high specific angular momentum to form accretion belts and launch jets. Say we take only parts with $j>6 \times 10^{14} \mathrm{~cm}^{2} \mathrm{~s}^{-1}$ according to Figure 6, the fluctuations in narrower shells are larger even. The mass in these parts is $\approx 0.1 M_{\odot}$. Accretion of this mass onto the NS of a mass of $\approx 2 M_{\odot}$, or later a $\mathrm{BH}$, can be channeled into jets with energy $\approx 10 \%$ of the rest mass of the shell. This amounts to $\approx 2 \times 10^{52} \mathrm{erg}$. To account for a CCSNe it is sufficient that only about 10 percent of the time the accretion belt/disk exists and launches jets (Papish \& Soker 2011). We emphasize that this last calculation is approximate and somewhat speculative. However, it is worth consideration as our main motivation for studying angular momentum fluctuations in the helium shell was implications for jet-driven explosion mechanisms. Due to the high resolution required, the need to explore convective regions closer to the iron-core, and the uncertainties of the jet-formation mechanism, we cannot yet reach definitive conclusions regarding the implications of convective regions for the jittering jets model.

\section{DISCUSSION AND SUMMARY}

We have presented a novel study of angular momentum fluctuations in stellar convective regions, using the sub-sonic solver MAESTRO. Further refinement and development of this method may enable the study of such fluctuations in different stellar models at different stages (e.g., just prior to corecollapse). These fluctuations may have implications on the core-collapse explosion mechanism, but may also be relevant for the process of stochastic spin-up of the core (Fuller et al. 2015). In the present study we found large-scale fluctuations of angular momentum in the convective helium zone of an evolved massive star, while the energy transport is similar to that expected from MLT, strengthening the validity of our simulation. Future studies will explore similar phenomena in silicon and oxygen shells (as done by, e.g., Mueller et al. 2016), and effects of rotation (as done by, e.g., Chatzopoulos et al. 2016).

In this study we focused on the convective helium zone surrounding an oxygen core. During the remainder of the stellar evolution time, the late burning stages eventually produce an iron core surrounded by silicon and oxygen shells. We assume that the structure of the helium shell does not change much in the relatively short time left to collapse. This implies that our results are relevant for the final stage of the star, although there may be latestage processes beyond our one-dimensional stellar modeling, such as significant wave-driven mass-loss (Ouataert \& Shiode 2012; Shiode \& Ouataert 2014) or expansion (Mcley \& Soker 2014).

The mass inner to the convective region studied here is 
$M_{\mathrm{in}} \approx 2.4 M_{\odot}$, and a jet-driven explosion powered by accretion from the helium zone will leave a heavy NS. Loss of mass-energy to escaping neutrinos may reduce the remnant mass to around $M_{\mathrm{NS}} \approx 2 M_{\odot}$. For a lower mass NS to form (e.g., with $M_{\mathrm{NS}} \approx 1.4 M_{\odot}$ ), a jet-driven explosion will have to be powered by accretion from the silicon/oxygen shells. For more massive stars, the helium shell may surround a core massive enough to form a BH upon collapse. If the accretion of helium powers an explosion, then the relevance of 'failed supernovae' (Kochanek et al. 2008; O'Connor \& Ott 2011; Kochanek 2014), or 'very weak supernovae' (Lovegrove \& Woosley 2013) may be lessened. A supernova leaving a BH remnant may be prevalent for very massive stars - this may be the case for, e.g., SN 2005gl (Gal-Yam \& Leonard 2009).

We can consider the present results on the source of stochastic accreted angular momentum from the helium shell in combination with our previous results on a possible stochastic angular momentum accretion from the silicon shell (Gilkis \& Soker 2015), and the results of Papish, Gilkis \& Soker (2015) regarding the stochastic angular momentum accretion as a result of the standing accretionshock instability (SASI). We then come at an emerging idea where the newly formed NS or BH accretes mass with notable specific angular momentum fluctuations. These accretion episodes might lead to the formation of intermittent accretion disks or accretion belts that launch jets (Schreier \& Soker 2016). The present study is another step in developing the jittering jets model for CCSNe. This is a preliminary study, and the presented results will have to be scrutinized with higherresolution simulations. Also, in future works we will need to consider the combined effect of the different sources of stochastic angular momentum, as well as the angular momentum contribution from rotation. Magnetic fields may also be relevant for the pre-collapse evolution of stochastic angular momentum, and they are most definitely important for the post-collapse possibility of jet formation.

\section{ACKNOWLEDGMENTS}

We thank an anonymous referee for helpful comments that improved the presentation of our results. We thank Michael Zingale for helpful correspondence regarding the MAESTRO code, as well as the rest of the developers of MAESTRO for making it accessible and publicly available for the scientific community. Simulations were ran on the Israeli astrophysics I-CORE astric HPC.

\section{APPENDIX}

\section{A. LOWER RESOLUTION SIMULATIONS}

To check the effects of the simulation resolution on the angular momentum fluctuations, we performed additional simulations with the best resolution of the AMR grid (termed finest-resolution) lower than our nominal simulation. Our computational resources prohibit us from increasing the resolution in a full simulation needed when looking at angular momentum of entire shells. Other properties of the flow are studied in higher resolution octant simulations. This is presented in Appendix B

Figure 8 shows the magnitude of the specific angular momentum in different shells for simulations of varying finest-resolutions. The behavior and magnitude are similar, although hard to quantify, due to the stochastic nature of the angular momentum in the shells. Figure 9 show the resolution dependency of the time-averaged value of the specific angular momentum in different shells, and of the time-averaged maximal Mach number. As in Figure 8, the effect of resolution on the specific angular momentum is not entirely clear. The maximal Mach number, however, varies very regularly with the changing resolution. Extrapolating linearly to infinite resolution reduces the Mach number by a factor of $\approx 2$ compared to our nominal simulation. We might expect the angular momentum to change accordingly, but this must be studied in simulations of higher resolution, beyond our current computational resources.

\section{B. OCTANT SIMULATIONS}

To further investigate the issue of convergence, we performed several simulations of one eighth of the full simulation with reflecting boundary conditions ('octant' simulations), with different maximal simulation resolutions. Two simulations, with finest resolutions of $800 \mathrm{~km}$ and $1250 \mathrm{~km}$, are presented at $t=20000 \mathrm{~s}$ in Figure 10. From Figure 10 we can see that the outer part of the convective region is similar in the two simulations, while in the inner part the simulation with lower finest-resolution the flow velocity is somewhat higher. We also see by comparing the upper to lower panel on each side that the magnitude of the radial velocity component and the magnitude of the circumferential velocity component are similar. This shows that the convective flow is well-developed.

Figure 11] shows the kinetic energy due to radial component of the velocity only and due to the circumferential component of the velocity only in the convective region, for octant simulations with three different finest-resolutions, as well as our nominal full simulation, at $t=20000 \mathrm{~s}$. The kinetic energy in the outer part seems converged, while the inner part is sensitive to the simulation resolution. The radial and circumferential flow components of the kinetic energy are nearly equal, correspondingly with the velocity flows seen in Figure 10,

Figure 12 shows the kinetic energy in the region between $r=40000 \mathrm{~km}$ and $r=280000 \mathrm{~km}$ as a function of simulation time. The kinetic energy in the simulations has a transient phase with a large value, and then goes down and plateaus. This behavior is similar to that obtained by Viallet et al. (2013) in their simulations of a convective envelope of a red giant star (see their Figure 2). It can be seen that the simulations are not converged, as the kinetic energy plateau is lower for more highly-resolved simulations, as is the time-averaged maximal Mach number. Extrapolating from the values of the maximal Mach number to infinite resolution reduces the Mach number by a factor of 2-3 compared to our nominal simulation, similar to the results of the full simulations with lower resolution discussed in Appendix $\mathrm{A}$

\section{REFERENCES}

Almgren, A. S., Bell, J. B., Rendleman, C. A., \& Zingale, M. 2006a, ApJ, 637,922
Almgren, A. S., Bell, J. B., Rendleman, C. A., \& Zingale, M. 2006b, ApJ, 649,927 

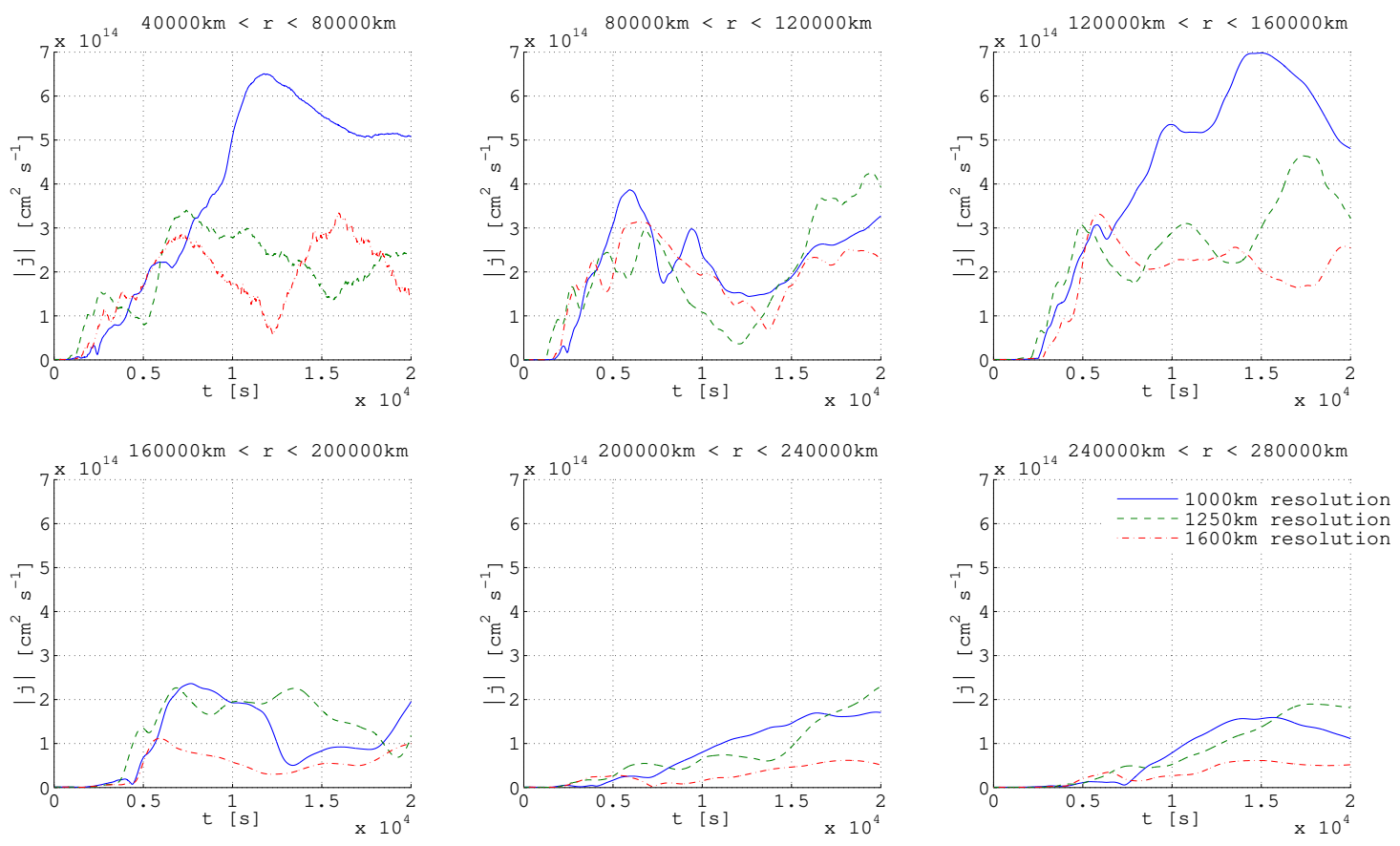

FIG. 8.- Specific angular momentum magnitude for different shells. The radial extent of the shells is detailed above each panel. Results from three different full-space simulations are shown, with finest-resolutions of $1000 \mathrm{~km}$ (the finest-resolution in our nominal simulation, analyzed in the main body of the paper), $1250 \mathrm{~km}$ and $1600 \mathrm{~km}$.
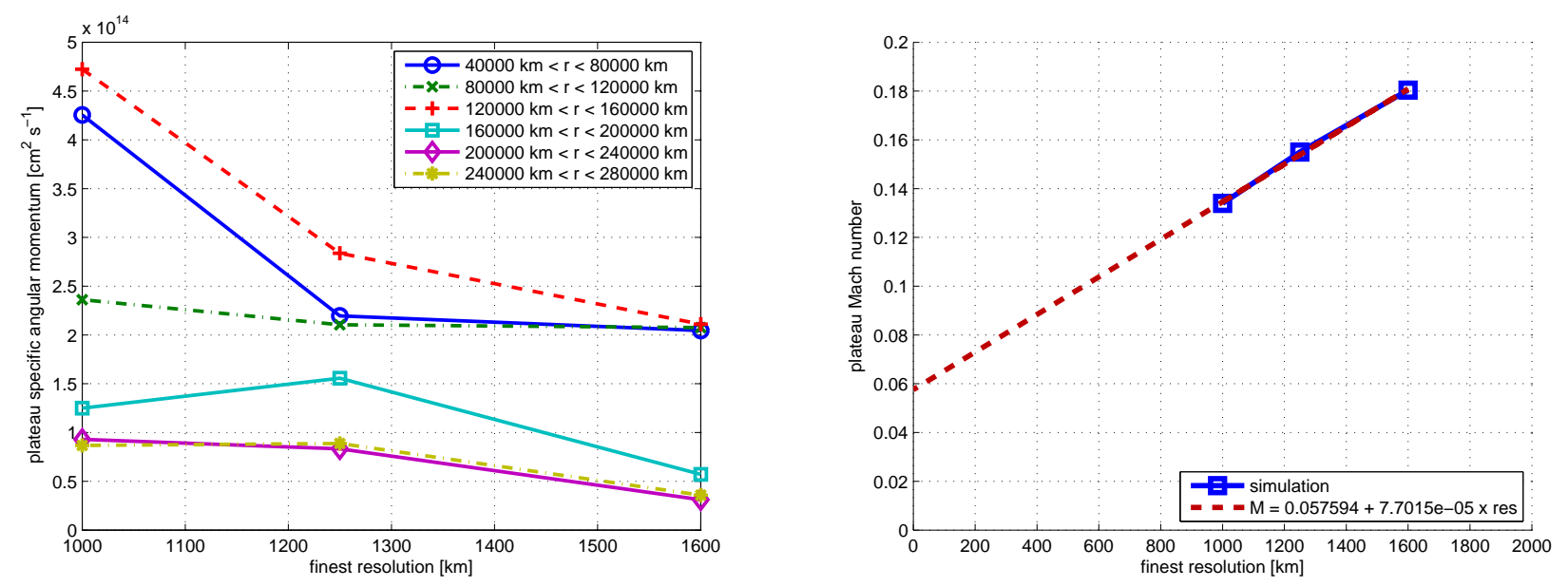

FIG. 9.- Left: Average over time (for $t>3000 \mathrm{~s}$ ) of the specific angular momentum in different shells for various finest-resolutions in full-space simulations. Right: Average value of the maximal Mach number for $t>3000 \mathrm{~s}$ as a function of finest grid resolution. The presented linear fit shows the extrapolated value of the Mach number, $\mathcal{M} \rightarrow 0.058$.

Almgren, A. S., Bell, J. B., Nonaka, A., \& Zingale, M. 2008, ApJ, 684, 449 Akiyama, S., Wheeler, J. C., Meier, D. L., Lichtenstadt, I. 2003, ApJ, 584, 954

Arnett, D., Meakin, C., Viallet, M., Campbell, S. W., Lattanzio, J. C., \& Mocák, M. 2015, ApJ, 809, 30

Arnett, D., Meakin, C., \& Young, P. A. 2009, ApJ, 690, 1715

Beeck, B., Schüssler, M., Cameron, R. H., \& Reiners, A. 2015, A\&A, 581, 42

Bethe, H. A., \& Wilson, J. R. 1985, ApJ, 295, 14

Blondin, J. M., \& Mezzacappa, A. 2007, Nature, 445, 58

Blondin, J. M., Mezzacappa, A., \& DeMarino, C. 2003, ApJ, 584, 971

Burrows, A., Hayes, J., \& Fryxell, B. A. 1995, ApJ, 450, 830

Chatzopoulos, E., Couch, S. M., Arnett, W. D., Timmes, F. X. 2016, ApJ, 822,61

Colgate, S. A., \& White, R. H. 1966, ApJ, 143, 626

Couch, S. M., Chatzopoulos, E., Arnett, W. D., \& Timmes, F. X. 2015, ApJ, $808, \mathrm{~L} 21$
Couch, S. M., \& Ott, C. D. 2013, ApJ, 778, L7

Couch, S. M., \& Ott, C. D. 2015, ApJ, 799, 5

Fernández, R. 2010, ApJ, 725, 1563

Fuller, J., Cantiello, M., Lecoanet, D., \& Quataert, E. 2015, ApJ, 810, 101

Gal-Yam, A., \& Leonard, D. C. 2009, Nature, 458, 865

Gilet, C., Almgren, A. S., Bell, J. B., Nonaka, A., Woosley, S. E., \& Zingale, M. 2013, ApJ, 773, 137

Gilkis, A. \& Soker, N. 2014, MNRAS, 439, 4011

Gilkis, A. \& Soker, N. 2015, ApJ, 806, 28

Janka, H.-T. 2012, Annual Review of Nuclear and Particle Science, 62, 407

Khokhlov, A. M., Höflich, P. A., Oran, E. S., et al. 1999, ApJ, 524, L107

Kippenhahn, R., Weigert, A., \& Weiss, A. 2012, Stellar Structure and

Evolution, Springer-Verlag Berlin Heidelberg

Kochanek, C. S. 2014, ApJ, 785, 28

Kochanek, C. S., Beacom, J. F., Kistler, M. D., Prieto, J. L., Stanek, K. Z.,

Thompson, T. A., \& Yüksel, H. 2008, ApJ, 684, 1336

Kushnir, D. 2015a, arXiv:1502.03111 


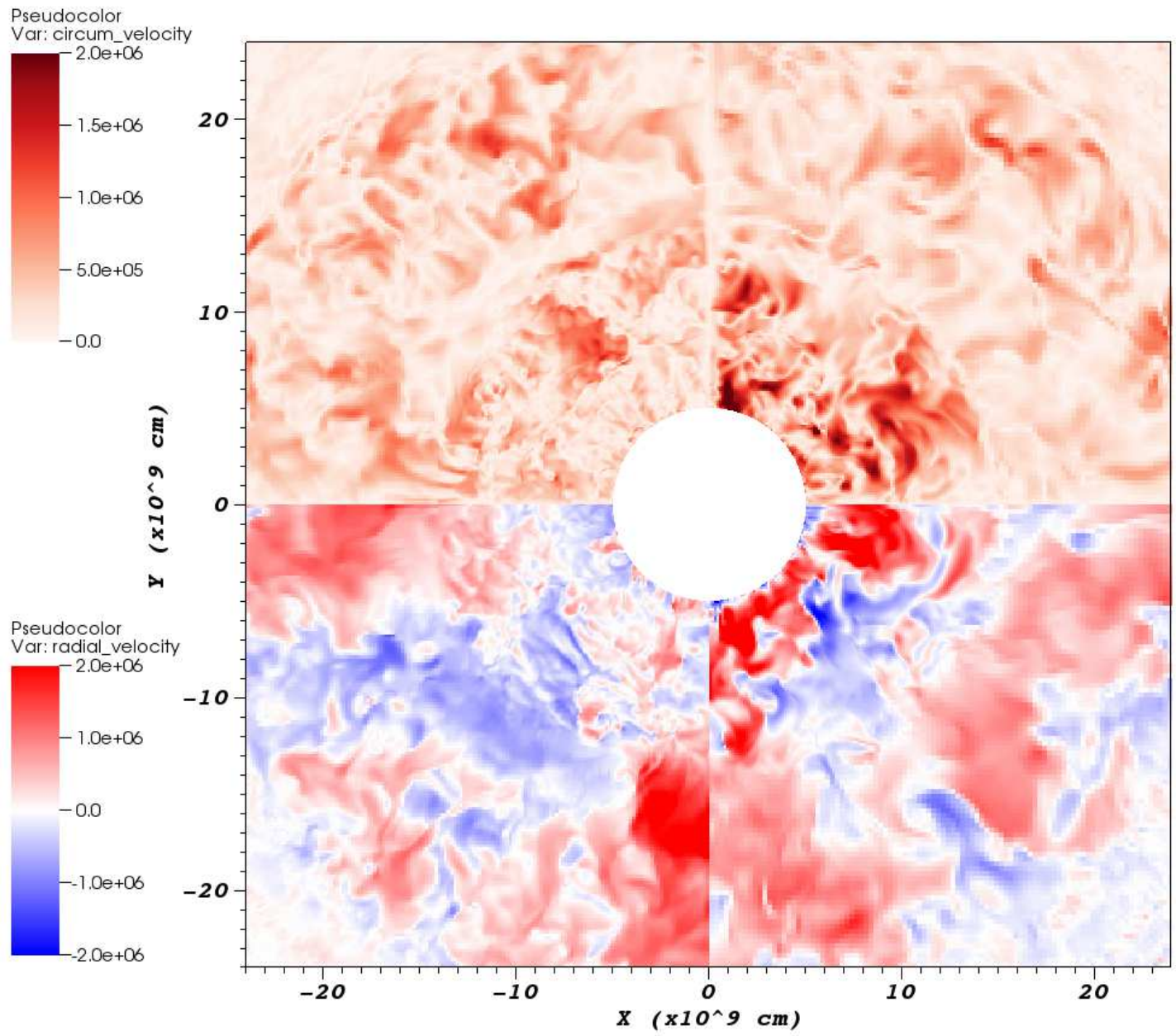

FIG. 10.- Presentation of two variables in two simulations in four different quarters. The two simulations are performed in one octant of the full space due to limited computer resources. All velocities are in units of $\mathrm{cm} \mathrm{s}^{-1}$ (so that the highest velocity magnitude presented is $2 \times 10^{6} \mathrm{~cm} \mathrm{~s}^{-1}$ ). Top: Circumferential velocity (i.e., perpendicular to the radial direction) with finest-resolution of $800 \mathrm{~km}$ (left) and $1250 \mathrm{~km}$ (right). Light shades of orange represent lower velocities, and darker shades represent high circumferential flow velocities. Bottom: Radial velocity with finest-resolution of $800 \mathrm{~km}$ (left) and $1250 \mathrm{~km}$ (right). Red shades represent outward motion, and blue shades represent inward motion. The inner oxygen core is omitted from the figure.

Kushnir, D. 2015b, arXiv:1506.02655

Kushnir, D., \& Katz, B. 2015, ApJ, 811, 97

Lazzati, D., Morsony, B. J., Blackwell, C. H., \& Begelman, M. C. 2012, ApJ, 750,68

LeBlanc, J. M., \& Wilson, J. R. 1970, ApJ, 161, 541

Liebendörfer, M., Rampp, M., Janka, H.-T., \& Mezzacappa, A. 2005, ApJ, 620,840

Lovegrove, E., \& Woosley, S. E. 2013, ApJ, 769, 109

Malone, C. M., Nonaka, A., Almgren, A. S., Bell, J. B., \& Zingale, M. 2011, ApJ, 728, 118

Malone, C. M., Zingale, M., Nonaka, A., Almgren, A. S., \& Bell, J. B. 2014, ApJ, 788, 115

Mcley, L., \& Soker, N. 2014, MNRAS, 445, 2492

Meakin, C. A., \& Arnett, D. 2007, ApJ, 667, 448

Mezzacappa, A., Liebendörfer, M., Messer, O. E., et al. 2001, Physical Review Letters, 86, 1935

Mösta, P., Ott, C. D., Radice, D., Roberts, L. F., Schnetter, E., Haas, R. 2015, Nature, 528, 376

Mueller, B., \& Janka, H.-T. 2015, MNRAS, 448, 2141

Mueller, B., Viallet, M., Heger, A., \& Janka, H.-T. 2016, arXiv:1605.01393

Nadezhin, D. K. 1980, Ap\&SS, 69, 115
Nonaka, A., Almgren, A. S., Bell, J. B., Lijewski, M. J., Malone, C. M., \& Zingale, M. 2010, ApJS, 188, 358

Nugis, T. \& Lamers, H. J. G. L. M. 2000, A\&A, 360, 227

O'Connor, E., \& Ott, C. D. 2011, ApJ, 730, 70

Papish, O., Gilkis, A., \& Soker, N. 2015, arXiv:1508.00218

Papish, O., Nordhaus, J., \& Soker, N. 2015, MNRAS, 448, 2362

Papish, O., \& Soker, N. 2011, MNRAS, 416, 1697

Papish, O., \& Soker, N. 2012a, in Roming P. W. A., Kawai N., Pian E., eds,

Proc. IAU Symp. 279, Death of Massive Stars: Supernovae and

Gamma-Ray Bursts. Cambridge Univ. Press, Cambridge, p. 377

Papish, O., \& Soker, N. 2012b, MNRAS, 421, 2763

Papish, O., \& Soker, N. 2014a, MNRAS, 438, 1027

Papish, O., \& Soker, N. 2014b, MNRAS, 443, 664

Paxton, B., Bildsten, L., Dotter, A., et al. 2011, ApJS, 192, 3

Paxton, B., Cantiello, M., Arras, P., et al. 2013, ApJS, 208, 4

Quataert, E., \& Shiode, J. 2012, MNRAS, 423, L92

Rampp, M., \& Janka, H.-T. 2000, ApJ, 539, L33

Schreier, R., \& Soker, N. 2016, RAA, 16e, 1

Shiode, J. H., \& Quataert, E. 2014, ApJ, 780, 96

Soker, N. 2010, MNRAS, 401, 2793

Viallet, M., Goffrey, T., Baraffe, I., Folini, D., Geroux, C., Popov, M. V., Pratt, J., \& Walder, R. 2016, A\&A, 586, 153 

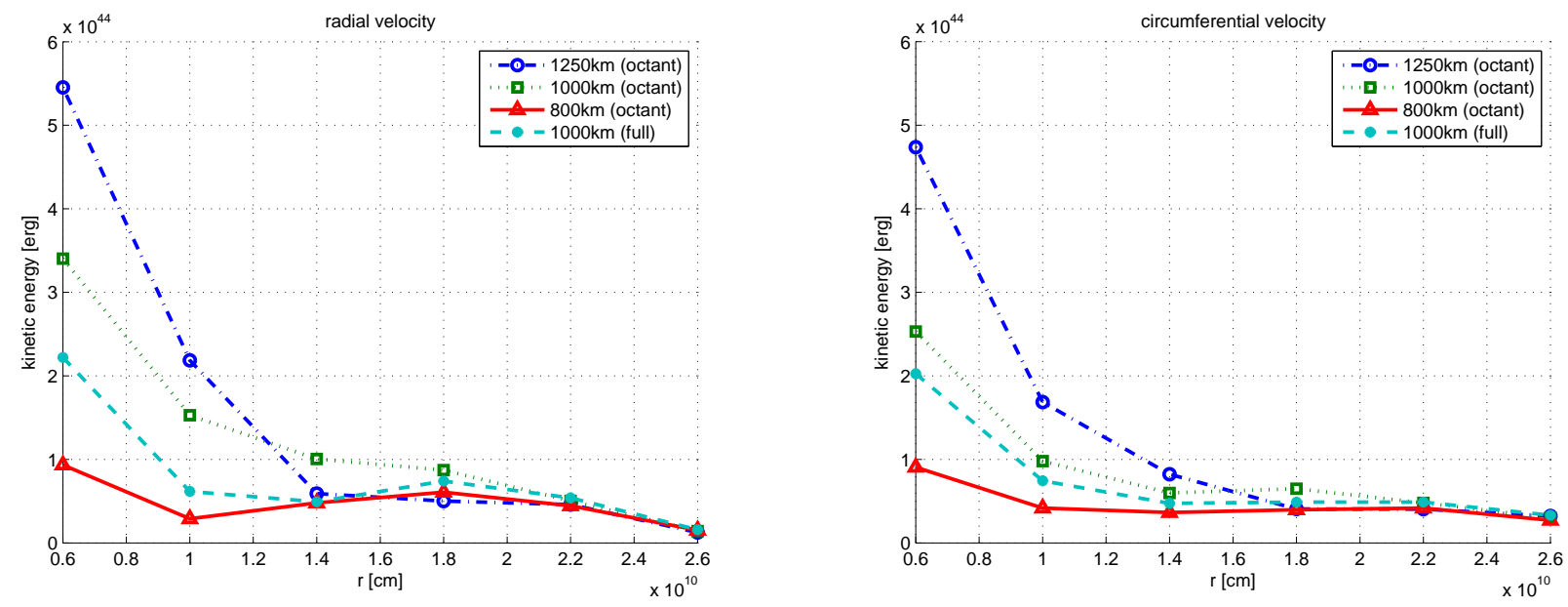

FIG. 11._Left: Kinetic energy of the radial velocity component for six different shells in octant simulations of varying finest resolution. The values for finest resolutions of $800 \mathrm{~km}$ and $1250 \mathrm{~km}$ are obtained for the simulations shown in Figure 10 The kinetic energy is multiplied by eight for the presentation of the octant simulations. Right: Kinetic energy of the circumferential velocity component (i.e., perpendicular to the radial direction) for six different shells in octant simulations of varying finest resolution. The $r$ coordinate is taken in the middle of each shell. The masses of the shells from the inner shell to the outer are 0.25 , $0.25,0.23,0.22,0.19$ and $0.17 M_{\odot}$.
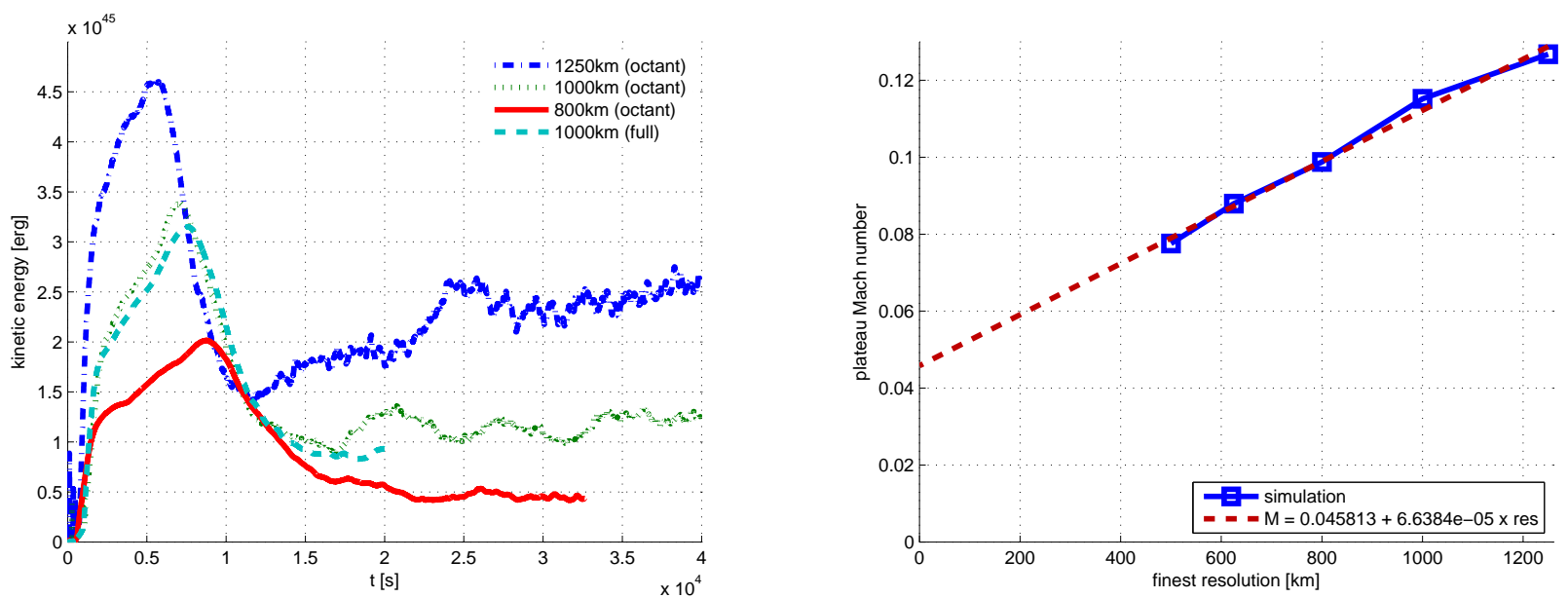

FIG. 12.- Left: Kinetic energy of the convective flow as function of time for our nominal simulation, and for three octant simulations with different resolutions (the kinetic energy is multiplied by eight for the presentation of the octant simulations). Right: Average value of the maximal Mach number for $t>3000 \mathrm{~s}$ as a function of finest grid resolution. The presented linear fit shows the extrapolated value of the Mach number, $\mathcal{M} \rightarrow 0.046$.

Viallet, M., Meakin, C., Arnett, D. \& Mocák, M. 2013, ApJ, 769, 1

Viallet, M., Meakin, C., Prat, V. \& Arnett, D. 2015, A\&A, 580, 61

Vink, J. S., de Koter, A., \& Lamers, H. J. G. L. M. 2001, A\&A, 369, 574
Zingale, M., Almgren, A. S., Bell, J. B., Nonaka, A., \& Woosley, S. E. 2009, ApJ, 704, 196

Zingale, M., Malone, C. M., Nonaka, A., Almgren, A. S., \& Bell, J. B. 2015, ApJ, 807, 60 